\title{
SIMULAÇÃO BASEADA EM AGENTES PARA AVALIAÇÃO DE INTERVENÇÕES NÃO-FARMACOLÓGICAS PARA O COMBATE À COVID-19 1
}

\author{
Thiago Augusto de Oliveira Silva ${ }^{\mathrm{a} *}$, Paganini Barcellos de Oliveira ${ }^{\mathrm{a}}$, Alexandre Xavier \\ Martins $^{\text {a }}$, Felipo Bacani ${ }^{\mathrm{b}}$ \\ a Instituto de Ciências Exatas e Aplicadas, Departamento de Engenharia de Produção \\ Universidade Federal de Ouro Preto, João Monlevade-MG, Brasil \\ ${ }^{\mathrm{b}}$ Instituto de Ciências Exatas e Aplicadas, Departamento de Ciências Exatas e Aplicadas \\ Universidade Federal de Ouro Preto, João Monlevade-MG, Brasil
}

Recebido 27/07/2020, aceito 23/10/2020

\begin{abstract}
RESUMO
Este trabalho propõe um modelo de simulação baseada em agentes capaz de avaliar os impactos de intervenções não-farmacológicas para o enfrentamento e controle da Covid-19. Políticas como o isolamento social e a realização de testes para detecção de infectados são estudadas, considerando os perfis probabilísticos de transmissão, morbidade e mortalidade. Os cenários simulados se baseiam em dados reais disponíveis na literatura, e as respostas do modelo são utilizadas para comparar o desempenho das alternativas, sob a ótica do total de infectados, o número básico de reprodução, o número de mortos e a duração da epidemia. Como resultado, verificou-se que a aplicação de isolamento horizontal é eficaz no combate a doença. $\mathrm{O}$ isolamento vertical, por outro lado, não apresentou desempenho satisfatório para os indicadores analisados. A saída antecipada do isolamento, bem como seu relaxamento e a abertura das fronteiras se demonstraram como principais entraves à contenção da doença.
\end{abstract}

Palavras-chave: Covid-19, Simulação baseada em agentes, Número básico de reprodução, Soluções não-farmacológicas.

\begin{abstract}
This paper proposes an agent-based simulation model to assess the impacts of different non-pharmacological interventions for coping and controlling the spread of the Covid-19 virus. Policies such as social isolation and testing for the detection of infected subjects are studied, considering the probabilistic profiles of transmission, morbidity, and mortality. The simulated scenarios are based on real data available in the literature, and the outputs of the model are used to compare the performance of the alternatives, from the perspective of the total infected, the basic reproductive number, the number of deaths and, the whole epidemic time. As a result, it was clear that horizontal social distancing is useful for combating the disease, while the vertical social distancing, did not perform satisfactorily. The early exit from the state of isolation or its relaxation, as well as the opening of borders, were the main obstacles to contain the disease.
\end{abstract}

Keywords: Covid-19, Agent-based simulation, Basic reproductive number, Non-pharmacological solutions.

\footnotetext{
*Autor para correspondência. E-mail: thiago@ufop.edu.br

DOI: 10.4322/PODes.2021.007
}

${ }^{1}$ Todos os autores assumem a responsabilidade pelo conteúdo do artigo. 


\section{Introdução}

O cenário pandêmico global, provocado pela disseminação do vírus da Covid-19, tem impulsionado o desenvolvimento de uma série de pesquisas científicas, nas mais variadas áreas do conhecimento, visando o desenvolvimento de políticas de enfrentamento e conscientização da população quanto à seleção de estratégias eficazes para o combate à doença (Lum e Tambyah, 2020). As áreas de ciências médicas, farmacêuticas e biológicas têm concentrado seus esforços na proposição de um conjunto de soluções farmacológicas que permitam tratar os pacientes ou eliminar/reduzir a chance de contágio (Ng et al., 2020; Lum e Tambyah, 2020; Sanders et al., 2020; Sahin et al., 2020).

De maneira complementar, Lana et al. (2020) afirmam que há um conjunto de soluções não-farmacológicas baseadas em dados reais, coletados, tratados matemática e estatisticamente e disponibilizados diariamente pelos agentes e órgãos de saúde de estados e municípios, que vêm sendo desenvolvidas por profissionais que atuam nas áreas de ciências exatas, sociais e da natureza. Tais soluções visam fornecer informações que ajudem na construção de políticas públicas, capazes de frear a disseminação da doença, bem como permitir o controle do número de leitos de enfermaria e UTI disponíveis para o tratamento dos infectados que necessitem de cuidados médicos especializados. Exemplos de soluções não-farmacológicas testadas e implantadas em diversos lugares do mundo todo, incluem medidas como: isolamento social, período de quarentena para os infectados, fechamento temporário de escolas, comércios e locais públicos (Agrawal et al., 2020). Em Candido et al. (2020) os autores apresentam uma análise dos efeitos de medidas não-farmacológicas de controle na difusão da Covid-19 no Brasil.

A operacionalização destas estratégias de enfrentamento dependem de um esforço conjunto do poder público e com a sociedade civil. Além disso, é importante que as estratégias propostas tenham embasamento científico suficiente para assegurar a efetividade da implantação de ações que cubram uma rede articulada de logística emergencial (Gagliano et al., 2020). A caracterização dessas ações e os desdobramentos de seus resultados podem ser feitas por meio de modelos matemáticos que simulem o comportamento da população e do vírus em cada localidade (Kucharski et al., 2020; Jewell et al., 2020).

A modelagem matemática de doenças infecciosas é frequentemente feita a partir de modelos compartimentais do tipo SIR, que subdividem toda a população em estudo em grupos como "suscetíveis", "infectados" e "recuperados". O modelo proposto neste trabalho inclui a subdivisão de "falecidos", logo faz parte da família de modelos SIRD (Vynnycky e White, 2010).

O procedimento de modelagem de ambientes, que sintetizam o comportamento de situações reais, exige um esforço significativo para a caracterização das particularidades e dos atributos correspondentes aos parâmetros e variáveis do problema (Wolfram, 2020; Cuevas, 2020; Riou e Althaus, 2020; Sanche et al., 2020; Wu et al., 2020). A determinação da proporção de infectados ao final de uma epidemia depende fortemente do número básico de reprodução, denotado por $\boldsymbol{R}_{\mathbf{0}}$. Este número representa quantas novas infecções um caso produziria em uma população composta apenas por indivíduos susceptíveis (Vynnycky e White, 2010, p. 77). Em outras palavras, este valor sintetiza o número médio de novas infecções de agentes saudáveis por agentes infectados em uma população, assim, quando $\boldsymbol{R}_{\mathbf{0}}>1$ há uma tendência de aumento no número de novos infectados (D'Arienzo e Coniglio, 2020). Por outro lado, o valor de $\boldsymbol{R}_{\mathbf{0}}$ pode variar em cada cenário estudado em função do comportamento dos agentes e das políticas de combate à disseminação do vírus, o que requer um tratamento adequado e individualizado no processo de estimativa deste e de outros parâmetros (Alimohamadi et al., 2020; Liu et al., 2020; D' Arienzo e Coniglio, 2020; You et al., 2020; Grassly e Fraser, 2008).

Em se tratando de estratégias de representação computacional para epidemias virais, a literatura mostra que existem diversas alternativas para modelagem de sistemas complexos capazes de representar o processo de disseminação de um vírus, tal como o Covid-19, incluindo modelos matemáticos (Lin et al., 2020; Chatterjee et al., 2020; Grassly e Fraser, 2008; Kissler et al., 2020), algoritmos de aprendizagem de máquina (Li et al., 2020a), modelos de simulação (Chan et al., 
2020) e regressões (Benvenuto et al., 2020), por exemplo. Uma destas alternativas trata-se de uma ferramenta numérica de representação computacional, também conhecida como Agent-Based Modeling and Simulation (ABMS) (Siegfried, 2014; Heppenstall et al., 2012; Taylor, 2014), que permite entender o comportamento de diversos agentes autônomos interagindo entre si, em um determinado ambiente, bem como, projetar, testar e avaliar diferentes tipos/níveis de intervenções no sistema, considerando os cenários e condições de interesse.

O grande potencial de flexibilidade da ABMS permite sua adaptação para diferentes contextos de disseminação de epidemias (Carpenter e Sattenspiel, 2009; Khalil et al., 2012; Adiga et al., 2018). Deste modo, sua aplicação se mostra eficiente quando se deseja analisar a dispersão de um vírus ao longo do tempo (Arifin et al., 2016). Estudos recentes têm mostrado que o cenário pandêmico em escala global, resultante do advento e disseminação do vírus da Covid-19, impulsionou e intensificou o uso do ABMS para avaliação de cenários (Ivanov, 2020; Ferguson et al., 2020; Wolfram, 2020; Klôh et al., 2020), considerando diferentes níveis de detalhamento e medidas de controle (econômicas, sanitárias e sociais) para o combate à proliferação da doença.

Em Wolfram (2020) é apresentado um modelo baseado em agentes para verificar como a interação entre eles influencia na disseminação da infecção. No entanto, o trabalho de Wolfram não considera a possibilidade de infecção pelo ambiente, ou seja, a transmissão do vírus ocorre apenas via outros agentes infectados, assim como a probabilidade de infecção é de $100 \%$ quando há o contato com infectados. Em Cuevas (2020) também é apresentada uma simulação baseada em agentes, mas dessa vez alterando-se as probabilidades de contágio de agente para agente. $\mathrm{O}$ objetivo neste caso era verificar como a infecção poderia se espalhar em uma fábrica, uma escola, etc.

Especificamente, neste trabalho, utiliza-se a metodologia ABMS para modelar os efeitos da evolução da pandemia de Covid-19 em uma determinada região geográfica, com uma densidade populacional pré-definida. Foram consideradas as formas de contágio propostas por Ferretti et al. (2020); Grassly e Fraser (2008). O trabalho de Ferretti et al. (2020) serviu como referência para a identificação dos parâmetros reais da Covid-19, tais como densidade do período de incubação (a partir de Lauer et al. (2020)), proporção de assintomáticos, infectividade de sintomáticos e assintomáticos, e a infectividade ambiental.

O modelo aqui desenvolvido também simula a introdução de medidas não-farmacológicas no ambiente simulado. Em MacIntyre e Chughtai (2020) e Cowling et al. (2020) os autores mostram como algumas das medidas aqui simuladas são efetivas no controle da Covid-19. Ademais, o estudo apresentado se difere de estudos similares (Ivanov, 2020; Ferguson et al., 2020; Wolfram, 2020; Klôh et al., 2020), pelos indicadores de análise utilizados e pelo foco no detalhamento da implementação das medidas não-farmacológicas.

Este trabalho está organizado conforme detalhado a seguir. A Seção 1 introduz e contextualiza o problema e a abordagem de resolução proposta. A Seção 2 apresenta o modelo de simulação, incluindo a caracterização dos agentes, das ações e das políticas de enfrentamento estudadas. A Seção 3 sumariza os resultados computacionais provenientes dos cenários simulados. Por fim, as principais conclusões e sugestões para trabalhos futuros são apresentados na Seção 4.

\section{Construção do Modelo}

O modelo de difusão do vírus Sars-CoV-2, causador da Covid-19, se baseia na interação de pessoas e entre pessoas e locais em uma comunidade. A doença é caracterizada pelos seguintes parâmetros: infectividade, período de incubação, duração da virose no hospedeiro, percentual de casos graves, chance de recuperação, percentual de pacientes que apresentam sintomas e o tempo de permanência do vírus no ambiente.

O mundo simulado foi construído considerando uma área de $10 \mathrm{~km}^{2}$ representados por 10.000 locais de $100 \mathrm{~m}^{2}$. O número de habitantes é definido com base no parâmetro "densidade demográfica" informado. Cada pessoa possui um local de residência que, em caso de isolamento 
social, só pode ser acessado por residentes. A quantidade de residências é definida pelo parâmetro "residentes por domicílio".

O sistema simulado é caracterizado pelo número inicial de casos e percentual da população no grupo de risco. O mundo, isto é, o ambiente simulado, é fechado, mas pode admitir a chegada de novos agentes provenientes do ambiente externo. O modelo considera que os doentes com casos graves, que não receberem atendimento especial, vão a óbito. Os doentes com casos graves que recebem atendimento se recuperam com probabilidade definida pelo parâmetro chance de recuperação. A capacidade de atendimento de casos graves do sistema local é um dado de entrada.

Os locais pertencentes ao ambiente simulado podem ser pontos de circulação ou residências, e podem ser infectados caso um agente tipo pessoa, infectada, transite por lá. A infectividade de um local é definida por uma função de densidade relacionada ao tempo de exposição do vírus no ambiente. A função de infectividade foi modelada de acordo com o proposto por Ferretti et al. (2020).

\subsection{Definição dos Agentes}

Os agentes são entidades autônomas de decisão, modeladas para acatar as medidas de segurança sanitárias vigentes. Durante o dia o agente se locomove aleatoriamente pelos pontos do mapa e pode ser infectado quando tem contato com algum doente ou com um local que possua o vírus. Uma vez infectado, o agente pode infectar outros agentes e locais.

A Figura 1 apresenta o diagrama simplificado de estados de um agente. Um agente pode ser saudável (S), pode estar infectado e ser assintomático $\left(I_{a}\right)$, pré-sintomático $\left(I_{p}\right)$ ou sintomático $\left(I_{s}\right)$. Uma vez doentes, os agentes podem se recuperar ficando imunes a novas contaminações $(R)$ ou vir a óbito $(M)$. A Tabela 1 descreve os parâmetros da Figura 1.

Figura 1: Diagrama de estados de um agente no sistema.

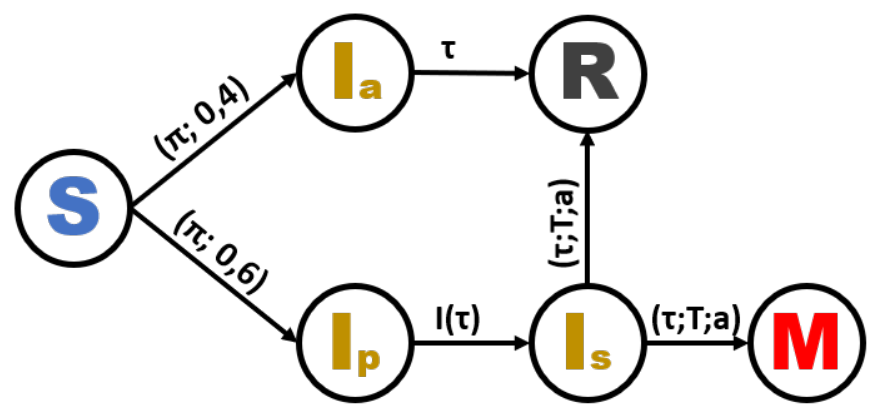

Fonte: Elaborado pelos autores.

Vale ressaltar que as interações com o ambiente e com outros agentes, a aplicação de políticas e o tempo são os fatores que determinam as transições entre os estados de um agente. Na Figura 1, a transição entre o estado $S$ e os estados $I_{a}$ e $I_{p}$ é afetada pelas políticas vigentes $(\pi)$, uma vez que, depende do contato com locais e outros agentes. Outro parâmetro determinante para a definição da transição entre $I_{a}$ e $I_{p}$ é a proporção de assintomáticos, fixada em 0,4 conforme sugerido por Ferretti et al. (2020). Um infectado pré-sintomático $\left(I_{p}\right)$ se torna sintomático $\left(I_{s}\right)$ após o período de incubação definido pela densidade de probabilidade $I(\tau)$ em relação ao tempo decorrido desde a contaminação $(\tau)$.

Infectados assintomáticos possuem versões mais brandas da doença e se recuperam após a duração da virose no organismo do hospedeiro. A depender do organismo e das características do agente $(a)$ e do tratamento recebido $(T)$ o infectado sintomático pode se recuperar ou vir a óbito. Além disso, definiu-se que a doença se manifesta de forma grave em $15 \%$ dos pacientes e que a taxa de mortalidade quando recebido tratamento adequado é de $5 \%$, uma estimativa otimista segundo Jung et al. (2020). Não foi identificado na literatura a possibilidade do infectado 
Tabela 1: Transição de estados dos agentes do modelo de simulação.

\begin{tabular}{ll}
\hline Símbolo & \multicolumn{1}{c}{ Descrição } \\
\hline Estados dos agentes \\
$S$ & Agente saudável. \\
$I_{a}$ & Infectado assintomático. \\
$I_{p}$ & Infectado pré-sintomático. \\
$I_{s}$ & Infectado sintomático. \\
$R$ & Agente recuperado e imune. \\
$M$ & Morto, em caso de falecimento do agente. \\
Parâmetro de transição \\
$\pi$ & Conjunto de políticas aplicadas. \\
$\tau$ & Tempo desde a infecção. \\
$I(\tau)$ & Densidade do período de incubação. \\
$T$ & Tratamento recebido pelo doente. \\
$a$ & Agente. \\
\hline \multicolumn{3}{c}{ Fonte: Elaborado pelos autores. }
\end{tabular}

assintomático vir a óbito, muito embora há evidências de implicações pulmonares não-letais, conforme $\mathrm{Hu}$ et al. (2020). Assim, embora possa haver a possibilidade de ocorrência desse evento em situações reais, essa particularidade foi desconsiderada na construção do modelo, o que pode ser entendido como uma simplificação do problema, quando a probabilidade de ocorrência do evento é muito baixa.

A cada dia, o agente poderá ou não circular no ambiente, a depender da política de isolamento vigente, de sua necessidade, e de seus atributos "grupo de risco" e "trabalhador essencial". Todo agente saudável está suscetível à infecção, mesmo em isolamento, que ocorre quando há contato com infectados ou locais onde infectados já estiveram. Caso seja sintomático, a depender da política, o paciente poderá ser colocado em isolamento onde aguardará a recuperação caso a doença não se agrave. Infectados circulantes assintomáticos podem ter a doença identificada por testagem, quando essa medida for implementada, e, neste caso, são postos em isolamento até se recuperar.

\subsection{Ações e Políticas}

De acordo com Valdez et al. (2013) a recorrência de doenças infecciosas, como as SRAGs (síndromes respiratórias agudas graves) e seu impacto cada vez maior na sociedade tem promovido o estudo de estratégias para frear o seu espalhamento. Muitos estudos teóricos são orientados para estabelecer como as estratégias podem manter o funcionamento da sociedade com o menor impacto econômico. Neste trabalho selecionou-se algumas destas estratégias para simular e entender o seu impacto no combate às doenças respiratórias, são elas:

- Isolamento Vertical: Nesta estratégia apenas alguns grupos são selecionados para estar em isolamento. Em geral, são grupos mais suscetíveis à infecção que está se espalhando no momento ou grupos com maior probabilidade de transmissão.

- Isolamento Horizontal: Na estratégia de isolamento horizontal todos os indivíduos da população devem se isolar independente da chance de desenvolver ou espalhar a doença.

- Isolamento de Sintomáticos: Nesta estratégia, como o próprio nome diz, a ideia é que os indivíduos da população sejam isolados a partir do momento que manifestarem os sintomas da doença.

- Testagem dos Circulantes: Esta estratégia se assemelha a anterior com a diferença que os indivíduos serão testados e poderão ser isolados caso estejam infectados, mesmo que não apresentem os sintomas. 


\subsection{Cálculo do Número Básico de Reprodução $R_{0}$}

Neste trabalho, a constante $\boldsymbol{R}_{\mathbf{0}}$ é calculada através do método "Exponential Growth" (EG) (Wallinga e Lipsitch, 2007), utilizando o pacote RO (em sua versão 1.2-6) do software R. O método $E G$ pressupõe que no início de toda epidemia há o crescimento exponencial do número de infectados. O coeficiente $\boldsymbol{R}_{\mathbf{0}}$ é obtido a partir da constante de crescimento exponencial do início da epidemia (que pode ser obtido por uma regressão linear, por exemplo) e do tempo de geração da doença, que representa a distribuição do intervalo de tempo entre um indivíduo ser infectado e chegar ao seu ponto de máxima infectividade (Last, 2001). O tempo de geração utilizado nas simulações é descrito por uma distribuição gama de média 7.5 e desvio padrão 3.5 ( $\mathrm{Li}$ et al., 2020b).

\section{Resultados}

O modelo computacional foi implementado no software Netlogo 6. A Figura 2 apresenta a tela do modelo no Netlogo. À esquerda são apresentados os comandos para ajuste dos parâmetros e políticas não-farmacológicas para o enfrentamento da doença, já ao centro, há uma tela que permite a animação dos agentes, o qual as casas são indicadas por uma coloração marrom e os agentes variam sua coloração dependendo do seu estado corrente (ver Tabela 1). Por fim, à direita, são apresentados os gráficos e indicadores que plotam a evolução da doença ao longo do tempo simulado.

Figura 2: Modelo implementado no software Netlogo.
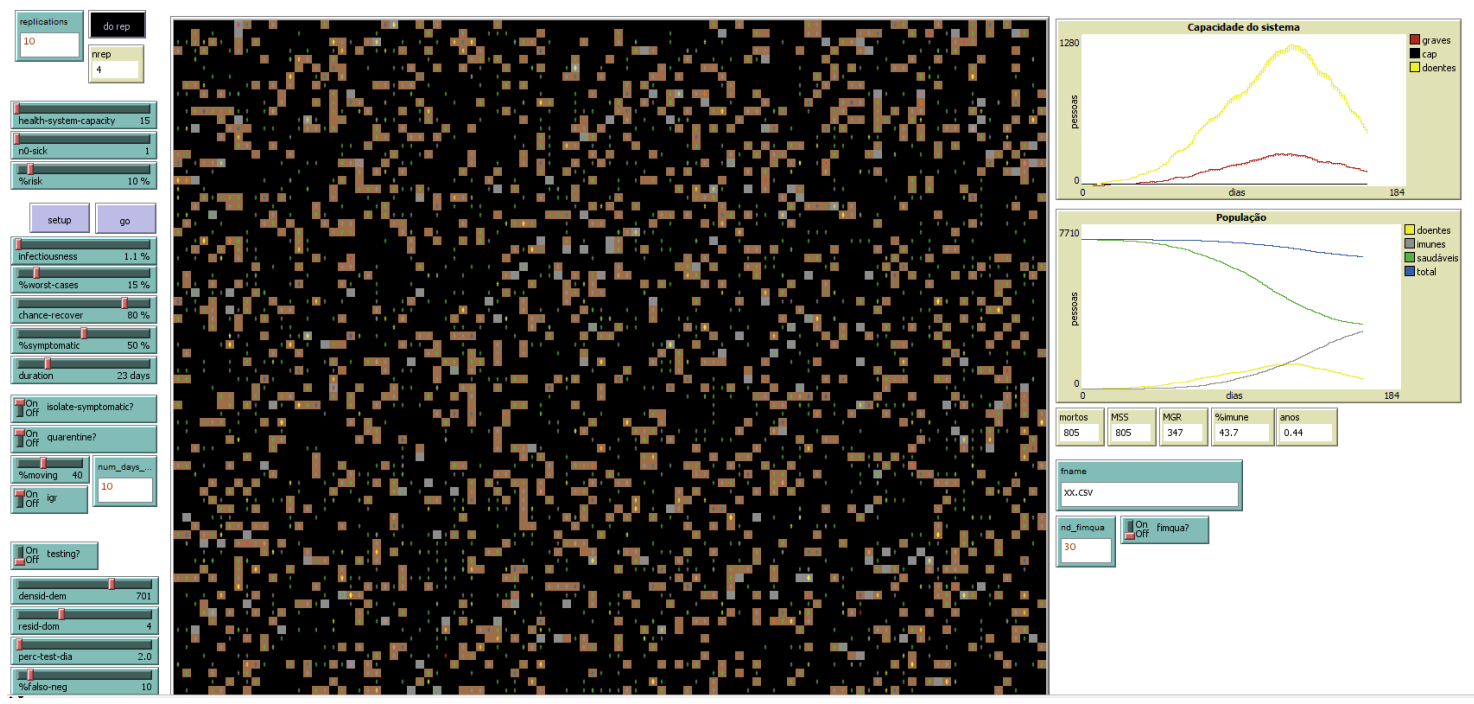

Fonte: Elaborado pelos autores.

\subsection{Definição de Cenários}

Para a realização dos experimentos computacionais simulados foram definidos um conjunto de cenários de interesse. Os cenários são baseados em estratégias adotadas por diversos órgãos governamentais de saúde no mundo, não necessariamente dissociadas ou exclusivas, visando combater eficientemente as SRAGs. A Tabela 2 sumariza a simbologia adotada para caracterização das diferentes ações não-farmacológicas adotadas em cada um dos cenários simulados.

A nomenclatura apresentada na Tabela 2 foi definida pensando nas diferentes possibilidades de implementação das medidas de combate epidemiológico, seja individual ou combinadas em um dado intervalo de tempo simulado. Assim, um hipotético cenário denominado $V T 0.1_{s}$ seria a combinação das ações V e T, com $0.1 \%$ da população testada diariamente, por exemplo. De acordo 
Tabela 2: Ações não-farmacológicas utilizadas nos cenários de teste.

\begin{tabular}{|c|c|c|}
\hline Símbolo & Descrição & Atributos das variações \\
\hline$N$ & Nenhuma medida de combate é implementada & - \\
\hline$V$ & Isolamento Vertical & - \\
\hline$H$ & Isolamento Horizontal & $\begin{array}{l}\text { \% da população circulando normalmente }(m) \\
\text { Tempo até o início do isolamento }(t) \\
\% \text { de redução da circulação de agentes } \\
\text { em isolamento }(f)\end{array}$ \\
\hline$A$ & Flexibilização antecipada das medidas & $\begin{array}{l}\text { \% da população circulando normalmente }(m) \\
\text { Tempo até o início do isolamento }(t) \\
\% \text { redução da circulação de agentes } \\
\text { em isolamento }(f) \\
\text { Tempo de isolamento }(d)\end{array}$ \\
\hline$T$ & Realização de um \% de testes na população & $\%$ da população testada diariamente $(s)$ \\
\hline
\end{tabular}

Fonte: Elaborado pelos autores.

com a Nota Técnica do Instituto de Estudos para Políticas de Saúde (Rache et al., 2020), o número de leitos no Brasil é em média de 15.6 leitos por 100 mil habitantes. Sendo assim, a capacidade de atendimento foi fixada em 15 indivíduos, simultaneamente, número mais que suficiente para o atendimento da população simulada em condições normais. Ademais, o tamanho da população é de 7010 habitantes. Um total de 19 diferentes cenários foram simulados, são eles:

- $N$, em que não se toma nenhuma medida de contenção;

- $T 0.1_{s}$, em que a porcentagem da população testada é $0.1 \%$;

- $V$, em que são isolados somente os indivíduos pertencentes ao grupo de risco;

- $H 5_{m} 10_{t}, H 10_{m} 10_{t}, H 20_{m} 10_{t}, H 30_{m} 10_{t}, H 40_{m} 10_{t}$ e $H 50_{m} 10_{t}$, em que o percentual de agentes circulando normalmente $(m)$ varia entre 5 e $50 \%$ com o isolamento social iniciando $(d) 10$ dias após o início da simulação. O padrão destes cenários é uma redução de $100 \%$ na movimentação dos agentes que não são circulantes $\left(100_{f}\right)$. Para simplificar a notação, essa informação foi omitida.

- $H 20_{m} 10_{t} 80_{f}$ e $H 20_{m} 10_{t} 60_{f}$, nos quais a redução da mobilidade dos agentes em isolamento $(f)$ é de $80 \%$ e $60 \%$;

- $H 20_{m} 30_{t}$, em que o isolamento começa apenas 30 dias após o início da simulação;

- $H T 20_{m} 10_{t} 60_{f} 0.1_{s}, H T 20_{m} 10_{t} 60_{f} 2.0_{s}$, em que são adotadas medidas de isolamento horizontal combinada com a testagem da população, com porcentagem de circulantes testados diariamente de $0.1 \%$ ou $2 \%$.

- $A 30_{m} 10_{t} 30_{d}, A 30_{m} 10_{t} 30_{d} 80_{f}$ e $A 30_{m} 10_{t} 30_{d} 60_{f}$, em que as medidas de isolamento são flexibilizadas antecipadamente, a porcentagem de agentes com circulação normal $(m)$ é de 30\%, o tempo até o início do isolamento $(t)$ é de 10 dias, a duração do isolamento $(d)$ é de 30 dias e a redução da mobilidade dos agentes em isolamento $(f)$ é de $100 \%, 80 \%$ e $60 \%$ respectivamente;

- $H 5_{m} 10_{t} E, \quad H 10_{m} 10_{t} E, \quad H 50_{m} 10_{t} E, \quad H T 20_{m} 10_{t} 60_{f} E, \quad H T 20_{m} 10_{t} 60_{f} 2_{s} E$, $H 20_{m} 10_{t} 80_{f} E, A 20_{m} 10_{t} 180_{d} 80_{f} E$ e $N E$, que são variações de alguns dos cenários descritos anteriormente, que incluem a simulação da chegada de visitantes do mundo exterior $(E)$ na cidade. A quantidade de visitante que chega diariamente é em média $0,15 \%$ da população e a probabilidade de estarem infectados é de $10 \%$.

Todos os cenários executados iniciam com 3 doentes e $2 \%$ da população pertencente ao grupo de risco. A doença simulada possui taxa básica de infecção de $4 \%$, que significa a chance de se infectar ao se encontrar com outro agente infectado, 3\% dos infectados apresentam quadros graves além do grupo de risco, $80 \%$ dos quadros graves se recuperam tendo acesso ao tratamento adequado, $60 \%$ dos infectados apresentam sintomas e a taxa de infecção de pessoas por ambientes 
contaminados é de $1 \%$. Contudo, pode-se dizer que se tratam de cenários conservadores em termos de mortalidade.

A densidade demográfica é de $701 \mathrm{hab} / \mathrm{km}^{2}$ e o número médio de residentes por domicílio é 4. Além disto, em caso de testagem, o teste tem resultado rápido e pode apresentar $10 \%$ de falso positivo e $20 \%$ de falso negativo. Para medir o desempenho de cada estratégia adotada foram utilizados três parâmetros, tempo até o fim da pandemia, número de infectados e número de mortos. Os cenários "Abertos", que permitem a entrada de agentes externos $(E)$, foram executados por 365 dias.

\subsection{Experimentos Computacionais}

Os experimentos foram realizados em um computador com 4 processadores AMD Ryzen $32200 \mathrm{G}$ de $3.5 \mathrm{GHz}$ e quatro núcleos cada, com 6GB de RAM e rodando sistema operacional Windows 10. Para cada cenário foram executadas 100 replicações e os dados analisados pelo software R.

As figuras 4, 5 e 6, apresentadas como apêndices, mostram a difusão esperada para todos os cenários simulados. São apresentados o número de casos (linha preta) e o número de casos graves (linha vermelha) esperados, por dia, ao longo da epidemia. O intervalo de confiança de $95 \%$ para média dos indicadores é apresentado através da região sombreada. A capacidade do sistema de tratar doentes graves é indicada pela linha azul tracejada.

A Tabela 3 apresenta as estatísticas dos cenários simulados. A primeira coluna indica o cenário simulado. A coluna seguinte se refere às estatísticas do número básico de reprodução dos cenários $\left(\overline{\boldsymbol{R}}_{0}\right)$. A coluna "Tempo (dias)", descreve a duração da epidemia, isto é, o tempo entre o primeiro caso e a eliminação total da doença. A quarta coluna, "\% de infec." se refere ao percentual da população que foi infectada pela doença, assim como a coluna subsequente "\% de mortos" se refere ao percentual de mortos. Para efeito de comparação, as estatísticas de tempo de duração da epidemia, percentual de infectados e percentual de mortos são comparadas de forma relativa nas colunas "Indicadores de desempenho". Quanto menor os valores destes indicadores, melhor o desempenho do cenário. Note que a Tabela 4 tem informações similares à Tabela 3, exceto pelo total de dias de simulação, tendo em vista que o tempo simulado, nestes casos, foram de 365 dias com possibilidade de entrada de agentes externos.

A Figura 3 apresenta o Boxplot do número básico de reprodução para cenários selecionados. É possível observar a variabilidade entre os cenários simulados, representados na figura como círculos, o que indica uma grande variabilidade do processo de difusão da doença.

Pela Tabela 3 pode-se notar que houve a contenção do número de casos da doença $\left(\overline{\boldsymbol{R}}_{\mathbf{0}}<1\right)$ nos cenários onde foi realizado:

- Isolamento horizontal onde a população em circulação é menor ou igual a $40 \%$, desde que seja implementada no curto prazo de 10 dias $\left(H 5_{m} 10_{t} / H 10_{m} 10_{t} / H 20_{m} 10_{t} /\right.$ $H 30_{m} 10_{t} / H 40_{m} 10_{t}$ ).

- Redução parcial da mobilidade dos agentes em isolamento, seja com a diminuição de $80 \%$ ou de $60 \%$ da circulação. No primeiro caso foi necessário realizar testes diários em $2 \%$ da população circulante $\left(H 20_{m} 10_{t} 80_{f} / H T 20_{m} 10_{t} 60_{f} 2.0_{s}\right)$.

- Isolamento horizontal com flexibilização antecipada após 30 dias apenas no caso onde houve total restrição à circulação da população circulante $\left(A 30_{m} 10_{t} 30_{d}\right)$.

Em se tratando dos cenários em que o número de casos da doença cresce $\left(\overline{\boldsymbol{R}}_{\mathbf{0}}>1\right)$, tabelas 3 e 4, as seguintes características são observadas:

- Tanto o isolamento vertical $(V)$ quanto a realização de testes em $0.1 \%$ da população $\left(T 0.1_{s}\right)$ não se mostraram políticas eficientes, apresentando, inclusive, valores de $\overline{\boldsymbol{R}}_{\mathbf{0}}$ similares ao do caso base $(N)$.

- Não implementar nenhuma política, seja no caso de ambiente aberto $(N)$ ou fechado $(N E)$, gerou $\overline{\boldsymbol{R}}_{\mathbf{0}}>1$, como esperado. O valor de $\overline{\boldsymbol{R}}_{\mathbf{0}}=1.55$ para $N$ teve valor abaixo do 
Figura 3: Boxplot do $\boldsymbol{R}_{\mathbf{0}}$.

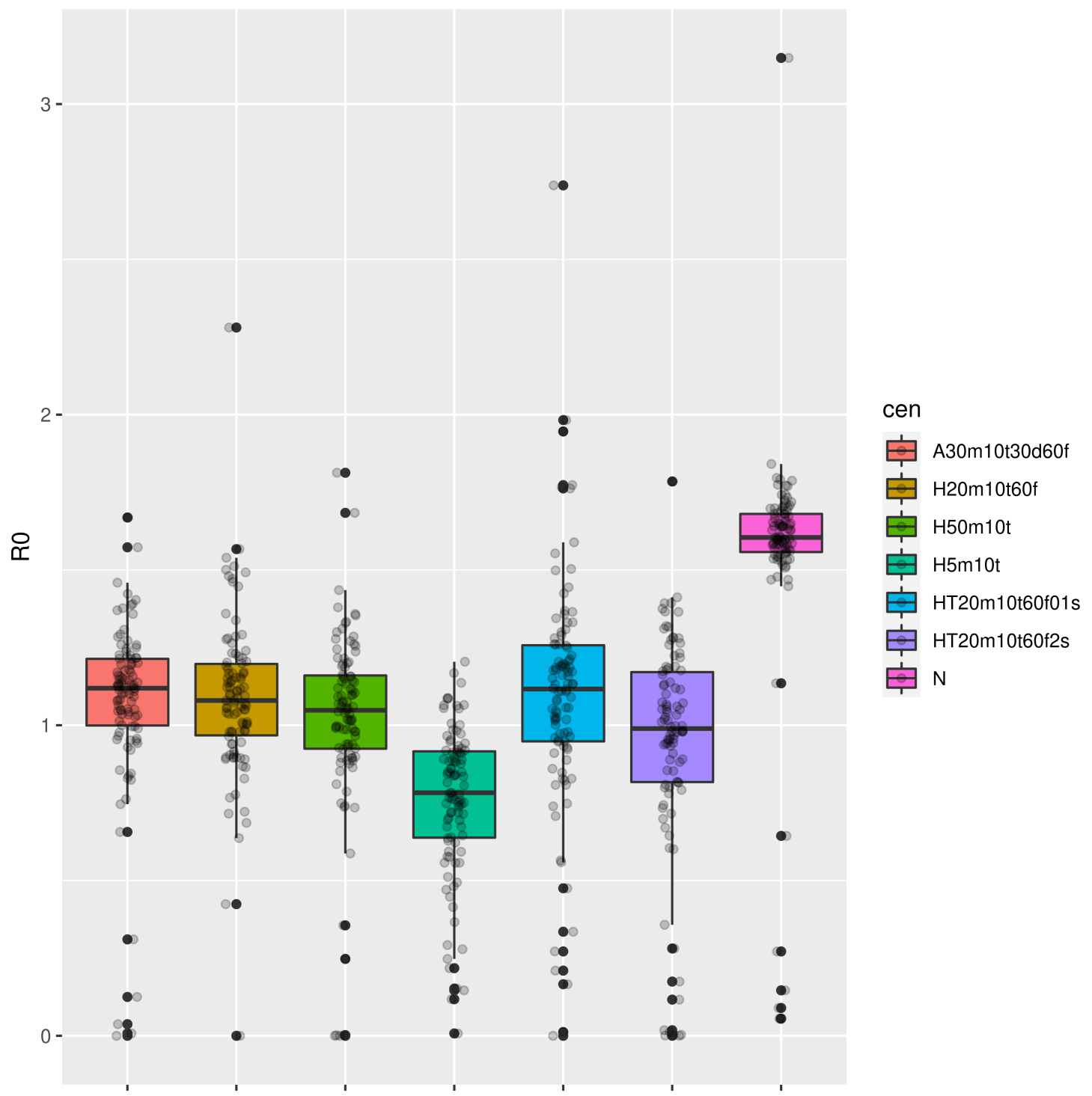

Fonte: Elaborado pelos autores.

valor de 1.94 à 5.70 que se observa na literatura (Li et al., 2020b; D'Arienzo e Coniglio, 2020; Riou e Althaus, 2020; Wu et al., 2020; Sanche et al., 2020). Podemos justificar esta disparidade pelo fato de um ambiente fechado ser uma situação que dificilmente se observa na prática.

Ainda pela Tabela 3 é possível perceber que não adotar nenhuma política $(N)$ não é a alternativa que implica no pior caso em termos de duração da epidemia, em média 84.97 dias. Por outro lado o cenário $N$ resulta no pior desempenho em termos de porcentagem de infectados e de mortos. Ao observar todos os indicadores de forma completa, verifica-se que o cenário $H 5_{m} 10_{t}$ obteve o melhor desempenho geral, o qual o tempo médio de duração da epidemia é de 35.1 dias e apenas $1.36 \%$ da população é infectada, em média, causando menos de $0,01 \%$ de mortes na população.

Considerando os indicadores de percentual de infectados, percentual de mortos e tempo de duração da epidemia, podemos notar que o isolamento horizontal $(H)$ foi eficiente em reduzir o número de óbitos e a duração da epidemia, mesmo com uma taxa elevada de circulantes $(50 \%)$. No entanto, nas instâncias onde a redução de mobilidade da população isolada cai para $80 \%$ e 
Tabela 3: Cenários simulados considerando população total de 7010 indivíduos e 100 replicações.

\begin{tabular}{|c|c|c|c|c|c|c|c|c|c|c|c|}
\hline \multirow{2}{*}{ Cenário } & \multicolumn{2}{|c|}{$\overline{\boldsymbol{R}}_{0}$} & \multicolumn{2}{|c|}{ Tempo (dias) } & \multicolumn{2}{|c|}{ \% de infec. } & \multicolumn{2}{|c|}{$\%$ de mortos } & \multicolumn{3}{|c|}{ Indicadores de desempenho* } \\
\hline & $\bar{x}$ & $\sigma$ & $\overline{\boldsymbol{x}}$ & $\sigma$ & $\bar{x}$ & $\sigma$ & $\overline{\boldsymbol{x}}$ & $\sigma$ & Tempo & Infectados & Mortes \\
\hline$A 30_{m} 10_{t} 30_{d}$ & 0.90 & 0.29 & 43.80 & 19.54 & 3.92 & 13.81 & 0.10 & 0.49 & 0.41 & 0.05 & 0.03 \\
\hline$A 30_{m} 10_{t} 30_{d} 60_{f}$ & 1.07 & 0.28 & 108.01 & 31.00 & 67.39 & 29.31 & 2.51 & 1.12 & 1.00 & 0.90 & 0.84 \\
\hline$A 30_{m} 10_{t} 30_{d} 80_{f}$ & 1.02 & 0.24 & 86.74 & 41.84 & 38.81 & 39.50 & 1.43 & 1.52 & 0.80 & 0.52 & 0.48 \\
\hline$H 5_{m} 10_{t}$ & 0.76 & 0.24 & 35.11 & 4.25 & 1.36 & 0.95 & 0.01 & 0.01 & 0.33 & 0.02 & $<0.01$ \\
\hline$H 10_{m} 10_{t}$ & 0.79 & 0.24 & 36.89 & 4.43 & 1.45 & 1.05 & 0.01 & 0.01 & 0.34 & 0.02 & $<0.01$ \\
\hline$H 20_{m} 10_{t}$ & 0.81 & 0.31 & 39.63 & 7.74 & 1.61 & 1.24 & 0.01 & 0.02 & 0.37 & 0.02 & $<0.01$ \\
\hline$H 30_{m} 10_{t}$ & 0.93 & 0.32 & 46.73 & 12.83 & 2.07 & 1.36 & 0.02 & 0.02 & 0.43 & 0.03 & $<0.01$ \\
\hline$H 40_{m} 10_{t}$ & 0.97 & 0.34 & 57.47 & 18.41 & 2.74 & 2.05 & 0.02 & 0.02 & 0.53 & 0.04 & $<0.01$ \\
\hline$H 50_{m} 10_{t}$ & 1.01 & 0.30 & 81.99 & 33.36 & 5.87 & 5.06 & 0.05 & 0.08 & 0.76 & 0.08 & 0.02 \\
\hline$H 20_{m} 30_{t}$ & 1.85 & 0.34 & 66.84 & 6.75 & 35.12 & 16.71 & 1.23 & 0.66 & 0.62 & 0.47 & 0.41 \\
\hline$H 20_{m} 10_{t} 60_{f}$ & 1.09 & 0.26 & 94.95 & 44.57 & 7.04 & 5.48 & 0.07 & 0.08 & 0.88 & 0.09 & 0.02 \\
\hline$H 20_{m} 10_{t} 80_{f}$ & 0.92 & 0.33 & 50.79 & 14.69 & 2.46 & 1.85 & 0.02 & 0.03 & 0.47 & 0.03 & $<0.01$ \\
\hline$H T 20_{m} 10_{t} 60_{f} 0.1_{s}$ & 1.09 & 0.39 & 86.03 & 38.84 & 6.00 & 4.76 & 0.06 & 0.08 & 0.80 & 0.08 & 0.02 \\
\hline$H T 20_{m} 10_{t} 60_{f} 2.0_{s}$ & 0.94 & 0.35 & 60.08 & 18.48 & 3.17 & 2.73 & 0.03 & 0.05 & 0.56 & 0.04 & $<0.01$ \\
\hline$N$ & 1.55 & 0.39 & 84.97 & 19.41 & 74.99 & 22.23 & 2.98 & 0.91 & 0.79 & 1.00 & 1.00 \\
\hline$T 0.1_{s}$ & 1.50 & 0.34 & 91.41 & 17.97 & 73.05 & 16.88 & 2.76 & 0.67 & 0.85 & 0.97 & 0.93 \\
\hline$V$ & 1.53 & 0.32 & 90.41 & 17.14 & 73.73 & 17.03 & 2.77 & 0.67 & 0.84 & 0.98 & 0.93 \\
\hline Máximo & 1.85 & 0.39 & 108.01 & 44.57 & 74.99 & 39.50 & 2.98 & 1.52 & & & \\
\hline Mínimo & 0.76 & 0.24 & 35.11 & 4.25 & 1.36 & 0.95 & 0.01 & 0.01 & & & \\
\hline
\end{tabular}

* Valores médios normalizados pelo pior caso ou $\left(\bar{x} / \bar{x}_{\text {máximo }}\right)$.

Fonte: Elaborado pelos autores.

$60 \%$ ( $H 20_{m} 10_{t} 80_{f}$ e $H 20_{m} 10_{t} 60_{f}$ ) percebe-se uma redução no desempenho dos indicadores. Neste sentido, é possível perceber que o desempenho é mais sensível à redução de mobilidade da população isolada $(f)$ do que ao percentual da população em isolamento $(m)$ para $m \leq 50$.

A saída do isolamento horizontal após 30 dias tornou ineficaz o esforço anterior para os cenários de $A 30_{m} 10_{t} 30_{d} 80_{f}$ e $A 30_{m} 10_{t} 30_{d} 60_{f}$ uma vez que a epidemia não havia sido contida no prazo. A testagem de circulantes $(t)$ só se demonstrou eficaz para o percentual de $2 \%$ de circulantes testados diariamente.

Observa-se também que, nos cenários em que há um ambiente aberto para entrada de agentes externos $(E)$, ver Tabela 4 , mesmo medidas de isolamento horizontal com a alta restrição de mobilidade (de 80\%) foram incapazes de impedir a expansão da doença. Cabe observar que, nestes cenários, não há controle sobre a epidemia mesmo após 365 dias. A falta de controle fica evidente quando analisados os resultados do cenário $A 30_{m} 10_{t} 180_{d} 80_{f}$ que simula a saída do isolamento após 180 dias. O desempenho deste cenário foi similar ao desempenho do cenário $N E$.

A série de dados do número de infectados ao longo do tempo nos cenários abertos, isto é, onde há chegada e saída constante de visitantes, dificultou o cálculo do $\overline{\boldsymbol{R}}_{\mathbf{0}}$ para esses cenários por apresentarem redução prematura no número de casos, devido à partida de visitantes infectados. $\mathrm{O}$ alto desvio padrão da estatística $\overline{\boldsymbol{R}}_{\mathbf{0}}$ dos casos $H 5_{m} 10_{t} E$ e $N E$ foi ocasionado pela existência de um outlier em uma das replicações de cada cenário onde o vetor de novos casos diários tem uma diminuição logo nos primeiros dias de epidemia, pelo fato de um agente infectado deixar o local e ainda não ter novos casos da doença. Este fato ocasionou grandes variações nos cálculos do $\boldsymbol{R}_{\mathbf{0}}$.

\section{Conclusão}

Este trabalho apresentou um modelo de simulação baseada em agentes capaz de simular, descrever e estimar o desempenho da combinação de diferentes medidas não-farmacológicas para 
Tabela 4: Cenários simulados considerando população total de 7010 indivíduos, 100 replicações, 365 dias de simulação e entrada de agentes externos $(E)$ ao longo do tempo.

\begin{tabular}{|c|c|c|c|c|c|c|c|c|}
\hline \multirow{2}{*}{ Cenário } & \multicolumn{2}{|c|}{$\overline{\boldsymbol{R}}_{0}$} & \multicolumn{2}{|c|}{$\%$ de infec. } & \multicolumn{2}{|c|}{$\%$ de mortos } & \multicolumn{2}{|c|}{ Indicadores de desempenho* } \\
\hline & $\overline{\boldsymbol{x}}$ & $\sigma$ & $\bar{x}$ & $\sigma$ & $\overline{\bar{x}}$ & $\sigma$ & Infectados & Mortes \\
\hline$H 5_{m} 10_{t} E$ & 2.93 & 17.57 & 11.54 & 5.56 & 0.16 & 0.19 & 0.14 & 0.05 \\
\hline$H 10_{m} 10_{t} E$ & 1.37 & 0.93 & 8.72 & 4.11 & 0.11 & 0.13 & 0.11 & 0.03 \\
\hline$H 50_{m} 10_{t} E$ & 2.12 & 7.90 & 28.90 & 6.63 & 0.48 & 0.41 & 0.36 & 0.14 \\
\hline$H T 20_{m} 10_{t} 60_{f} E$ & 1.16 & 0.30 & 32.36 & 6.69 & 0.54 & 0.38 & 0.40 & 0.16 \\
\hline$H T 20_{m} 10_{t} 60_{f} 2_{s} E$ & 1.40 & 2.36 & 44.12 & 21.17 & 1.36 & 0.89 & 0.55 & 0.40 \\
\hline$H 20_{m} 10_{t} 80_{f} E$ & 1.75 & 4.53 & 17.69 & 7.87 & 0.29 & 0.32 & 0.22 & 0.09 \\
\hline$A 20_{m} 10_{t} 180_{d} 80_{f} E$ & 1.17 & 0.36 & 76.51 & 2.80 & 2.81 & 0.34 & 0.95 & 0.83 \\
\hline$N E$ & 5.89 & 40.01 & 80.56 & 1.07 & 3.38 & 0.19 & 1.00 & 1.00 \\
\hline Máximo & 5.89 & 40.01 & 80.56 & 21.17 & 3.38 & 0.89 & & \\
\hline Mínimo & 1.16 & 0.30 & 8.72 & 1.07 & 0.11 & 0.13 & & \\
\hline
\end{tabular}

* Valores médios normalizados pelo pior caso ou $\left(\bar{x} / \bar{x}_{\text {máximo }}\right)$.

Fonte: Elaborado pelos autores.

o controle da disseminação do vírus da Covid-19.

Os resultados dos testes realizados mostraram a importância das medidas de isolamento social e de testagem por amostragem para a minimização do número de óbitos, para minimização da duração da circulação do vírus e redução do número básico de reprodução, resultado consistente com o apresentado por Candido et al. (2020).

Um outro aspecto relevante a ser considerado é a incapacidade de controle das epidemias em cenários abertos, o que indica que é necessário uma ação coordenada de diversas localidades para que as medidas implementadas em cada local sejam efetivas.

O modelo proposto se mostra sensível aos parâmetros de entrada e se comporta como o esperado, de acordo com a literatura. Isto é, demonstra que as políticas não-farmacológicas de isolamento social são eficazes no que tange o combate à disseminação do vírus e que quanto maior grau de isolamento, maior é a sua a eficiência como política de enfrentamento (Cowling et al., 2020). A conclusão a respeito da eficácia de uma medida não pode ser generalizada para diferentes localidades. Neste sentido, o desenvolvimento de uma ferramenta de simulação é uma contribuição para a análise customizada de cada caso.

Pode-se, entretanto, generalizar a dominância entre as medidas. O comportamento exponencial de crescimento do número de casos está bem representado, tal como o efeito que as medidas não-farmacológicas possuem sobre o crescimento.

Sabe-se, no entanto, que a realidade se dará por uma mudança constante das aplicações de medidas não-farmacológicas de controle, tendo como referência a resposta em termos do desempenho, isto é, a aplicação de tais medidas se dará de forma dinâmica ao longo da duração da pandemia. Como limitações do trabalho, o modelo poderia considerar os fluxos de deslocamento de forma mais realista. Outras medidas de controle como barreira sanitária e medição de temperatura em massa poderiam, também, ser consideradas.

Em trabalhos futuros pretende-se construir um modelo mais completo, hierárquico, para simular grandes regiões geográficas. Pretende-se combinar Simulação de Monte Carlo, Simulação Baseada em Agentes, Aprendizado de Máquina e Sistemas de Informações Geográficas (SIGs) com foco na disseminação e permanência do vírus no ambiente por meio de dados reais da propagação de SRAGs, dados demográficos, socioeconômicos, sanitários e da capacidade do sistema de saúde de uma determinada região.

Agradecimentos. Os autores gostariam de agradecer à CAPES, ao CNPq, à FAPEMIG e à UFOP pelo apoio ao desenvolvimento deste projeto e aos avaliadores pelas contribuições. 


\section{Referências}

Adiga, A., Chu, S., Eubank, S., Kuhlman, C. J., Lewis, B., Marathe, A., et al. Disparities in spread and control of influenza in slums of Delhi: findings from an agent-based modelling study. $B M J$ Open, v. 8, n. 1, e017353, 2018. https://doi.org/10.1136/bmjopen-2017-017353.

Agrawal, S., Goel, A. D., e Gupta, N. Emerging prophylaxis strategies against COVID-19. Monaldi Archives for Chest Disease, v. 90, n. 1, p. 169-172, 2020. https://doi.org/10.4081/monaldi.2020.1289.

Alimohamadi, Y., Taghdir, M., e Sepandi, M. Estimate of the basic reproduction number for COVID-19: A systematic review and meta-analysis. Journal of Preventive Medicine \& Public Health, v. 53, n. 3, p. 151-157, 2020. https://doi.org/10.3961/jpmph.20.076.

Arifin, S. M. N., Madey, G. R., e Collins, F. H. Spatial Agent-Based Simulation Modeling in Public Health: Design, Implementation, and Applications for Malaria Epidemiology. New Jersey: Wiley, 2016.

Benvenuto, D., Giovanetti, M., Vassallo, L., Angeletti, S., e Ciccozzi, M. Application of the ARIMA model on the COVID-2019 epidemic dataset. Data in Brief, v. 29, 105340, 2020. https://doi.org/10.1016/j.dib.2020.105340.

Candido, D. S., Claro, I. M., de Jesus, J. G., Souza, W. M., Moreira, F. R. R., Dellicour, S., et al. Evolution and epidemic spread of SARS-CoV-2 in Brazil. Science, v. 369, n. 6508, p. 1255-1260, 2020. https://doi.org/10.1126/science.abd2161.

Carpenter, C. e Sattenspiel, L. The design and use of an agent-based model to simulate the 1918 influenza epidemic at Norway House, Manitoba. American Journal of Human Biology, v. 21, n. 3, p. 290-300, 2009. https://doi.org/10.1002/ajhb.20857.

Chan, J. F.-W., Zhang, A. J., Yuan, S., Poon, V. K.-M., Chan, C. C.-S., Lee, A. C.-Y., et al. Simulation of the clinical and pathological manifestations of Coronavirus Disease 2019 (COVID-19) in golden Syrian hamster model: implications for disease pathogenesis and transmissibility. Clinical Infectious Diseases, v. 71, n. 9, p. 2428-2446, 2020. https://doi.org/10.1093/cid/ciaa325.

Chatterjee, K., Chatterjee, K., Kumar, A., e Shankar, S. Healthcare impact of COVID-19 epidemic in India: A stochastic mathematical model. Medical Journal Armed Forces India, v. 76, n. 2, p. 147-155, 2020. https://doi.org/10.1016/j.mjafi.2020.03.022.

Cowling, B. J., Ali, S. T., Ng, T. W. Y., Tsang, T. K., Li, J. C. M., Fong, M. W., et al. Impact assessment of non-pharmaceutical interventions against coronavirus disease 2019 and influenza in Hong Kong: an observational study. The Lancet Public Health, v. 5, n. 5, p. e279-e288, 2020. https://doi.org/10.1016/S2468-2667(20)30090-6.

Cuevas, E. An agent-based model to evaluate the COVID-19 transmission risks in facilities. Computers in Biology and Medicine, v. 121, 103827, 2020. https://doi.org/10.1016/j.compbiomed.2020.103827.

D'Arienzo, M. e Coniglio, A. Assessment of the SARS-CoV-2 basic reproduction number, $R_{0}$, based on the early phase of COVID-19 outbreak in Italy. Biosafety and Health, v. 2, n. 2, p. 57-59, 2020. https://doi.org/10.1016/j.bsheal.2020.03.004.

Ferguson, N. M., Laydon, D., Nedjati-Gilani, G., Imai, N., Ainslie, K., Baguelin, M., et al. Impact of non-pharmaceutical interventions (NPIs) to reduce covid19 mortality and healthcare demand. Report 9, Imperial College COVID-19 Response Team London, UK , 2020. p. 1-20. https://doi.org/10.25561/77482. 
Ferretti, L., Wymant, C., Kendall, M., Zhao, L., Nurtay, A., Abeler-Dörner, L., et al. Quantifying SARS-CoV-2 transmission suggests epidemic control with digital contact tracing. Science, v. 368, n. 6491, eabb6936, 2020. https://doi.org/10.1126/science.abb6936.

Gagliano, A., Villani, P. G., Co', F. M., Manelli, A., Paglia, S., Bisagni, P. A. G., et al. COVID-19 epidemic in the middle province of Northern Italy: Impact, logistics, and strategy in the first line hospital. Disaster Medicine and Public Health Preparedness, v. 14, n. 3, p. 372-376, 2020. https://doi.org/10.1017/dmp.2020.51.

Grassly, N. C. e Fraser, C. Mathematical models of infectious disease transmission. Nature Reviews Microbiology, v. 6, n. 6, p. 477-487, 2008.

Heppenstall, A., Crooks, A., See, L. M., e Batty, M. (eds.). Agent-Based Models of Geographical Systems. $1^{\mathrm{a}}$ ed. Dordrecht: Springer Netherlands, 2012.

Hu, Z., Song, C., Xu, C., Jin, G., Chen, Y., Xu, X., et al. Clinical characteristics of 24 asymptomatic infections with COVID-19 screened among close contacts in Nanjing, China. Science China Life Sciences, v. 63, n. 5, p. 706-711, 2020. https://doi.org/10.1007/s11427-020-1661-4.

Ivanov, D. Predicting the impacts of epidemic outbreaks on global supply chains: A simulation-based analysis on the coronavirus outbreak (COVID-19/SARS-CoV-2) case. Transportation Research Part E: Logistics and Transportation Review, v. 136, 101922, 2020. https://doi.org/10.1016/j.tre.2020.101922.

Jewell, N. P., Lewnard, J. A., e Jewell, B. L. Predictive Mathematical Models of the COVID-19 Pandemic: Underlying Principles and Value of Projections. JAMA, v. 323, n. 19, p. 1893-1894, 2020. https://doi.org/10.1001/jama.2020.6585.

Jung, S.-m., Akhmetzhanov, A. R., Hayashi, K., Linton, N. M., Yang, Y., Yuan, B., et al. Real-time estimation of the risk of death from novel coronavirus (COVID-19) infection: Inference using exported cases. Journal of Clinical Medicine, v. 9, n. 2, 523, 2020. https://doi.org/10.3390/jcm9020523.

Khalil, K. M., Abdel-Aziz, M., Nazmy, T. T., e Salem, A.-B. M. An agent-based modeling for pandemic influenza in egypt. In: Lu, J., Jain, L. C., e Zhang, G. (eds.), Handbook on Decision Making: Vol 2: Risk Management in Decision Making, Vol. 33, p. 205-218. Berlin, Heidelberg: Springer Berlin Heidelberg, 2012.

Kissler, S. M., Tedijanto, C., Lipsitch, M., e Grad, Y. H. Social distancing strategies for curbing the COVID-19 epidemic. medRxiv, 2020. https://doi.org/10.1101/2020.03.22.20041079.

Klôh, V. P., Silva, G. D., Ferro, M., Araújo, E., de Melo, C. B., de Andrade Lima, J. R. P., e Martins, E. R. The virus and socioeconomic inequality: An agent-based model to simulate and assess the impact of interventions to reduce the spread of COVID-19 in Rio de Janeiro, Brazil. Brazilian Journal of Health Review, v. 3, n. 2, p. 647-3673, 2020. https://doi.org/10.34119/bjhrv3n2-192.

Kucharski, A. J., Russell, T. W., Diamond, C., Liu, Y., Edmunds, J., Funk, S., et al. Early dynamics of transmission and control of COVID-19: a mathematical modelling study. The Lancet Infectious Diseases, v. 20, n. 5, p. 553-558, 2020. https://doi.org/10.1016/S1473-3099(20)30144-4.

Lana, R. M., Coelho, F. C., Gomes, M. F. d. C., Cruz, O. G., Bastos, L. S., Villela, D. A. M., e Codeço, C. T. Emergência do novo coronavírus (SARS-CoV-2) e o papel de uma vigilância nacional em saúde oportuna e efetiva. Cadernos de Saúde Pública, v. 36, n. 3, e00019620, 2020. https://doi.org/10.1590/0102-311x00019620. 
Last, J. M., (ed.). A Dictionary of Epidemiology. $4^{\text {a }}$ ed. New York: Oxford University Press, 2001.

Lauer, S. A., Grantz, K. H., Bi, Q., Jones, F. K., Zheng, Q., Meredith, H. R., et al. The incubation period of coronavirus disease 2019 (COVID-19) from publicly reported confirmed cases: estimation and application. Annals of Internal Medicine, v. 172, n. 9, p. 577-582, 2020.

Li, L., Qin, L., Xu, Z., Yin, Y., Wang, X., Kong, B., et al. Using Artificial Intelligence to Detect COVID-19 and Community-acquired Pneumonia Based on Pulmonary CT: Evaluation of the Diagnostic Accuracy. Radiology, v. 296, n. 2, p. E65-E71, 2020a. https://doi.org/10.1148/radiol.2020200905.

Li, Q., Guan, X., Wu, P., Wang, X., Zhou, L., Tong, Y., et al. Early transmission dynamics in Wuhan, China, of novel coronavirus-infected pneumonia. New England Journal of Medicine, v. 382, n. 13, p. 1199-1207, 2020b. https://doi.org/10.1056/NEJMoa2001316.

Lin, Q., Zhao, S., Gao, D., Lou, Y., Yang, S., Musa, S. S., et al. A conceptual model for the coronavirus disease 2019 (COVID-19) outbreak in Wuhan, China with individual reaction and governmental action. International Journal of Infectious Diseases, v. 93, p. 211-216, 2020. https://doi.org/10.1016/j.ijid.2020.02.058.

Liu, Y., Gayle, A. A., Wilder-Smith, A., e Rocklöv, J. The reproductive number of COVID-19 is higher compared to SARS coronavirus. Journal of Travel Medicine, v. 27, n. 2, taaa021, 2020. https://doi.org/10.1093/jtm/taaa021.

Lum, L. H. W. e Tambyah, P. A. Outbreak of COVID-19 - an urgent need for good science to silence our fears? Singapore Medical Journal, v. 61, n. 2, p. 55-57, 2020. https://doi.org/10.11622/smedj.2020018.

MacIntyre, C. R. e Chughtai, A. A. A rapid systematic review of the efficacy of face masks and respirators against coronaviruses and other respiratory transmissible viruses for the community, healthcare workers and sick patients. International Journal of Nursing Studies, v. 108, 103629, 2020. https://doi.org/10.1016/j.ijnurstu.2020.103629.

Ng, M.-Y., Lee, E. Y. P., Yang, J., Yang, F., Li, X., Wang, H., et al. Imaging profile of the COVID-19 infection: Radiologic findings and literature review. Radiology: Cardiothoracic Imaging, v. 2, n. 1, e200034, 2020. https://doi.org/10.1148/ryct.2020200034.

Rache, B., Rocha, R., Nunes, L., Spinola, P., Malik, A. M., e Massuda, A. Necessidades de Infraestrutura do SUS em Preparo ao COVID-19: Leitos de UTI, Respiradores e Ocupação Hospitalar. Nota Técnica, n.3, IEPS, São Paulo, 2020.

Riou, J. e Althaus, C. L. Pattern of early human-to-human transmission of Wuhan 2019 novel coronavirus (2019-nCoV), December 2019 to January 2020. Eurosurveillance, v. 25, n. 4, 2000058, 2020. https://doi.org/10.2807/1560-7917.ES.2020.25.4.2000058.

Sahin, A. R., Erdogan, A., Agaoglu, P. M., Dineri, Y., Cakirci, A. Y., Senel, M. E., et al. 2019 novel coronavirus (COVID-19) outbreak: A review of the current literature. EJMO, v. 4, n. 1, p. 1-7, 2020. https://doi.org/10.14744/ejmo.2020.12220.

Sanche, S., Lin, Y. T., Xu, C., Romero-Severson, E., Hengartner, N., e Ke, R. High contagiousness and rapid spread of severe acute respiratory syndrome coronavirus 2. Emerging Infectious Diseases, v. 26, n. 7, p. 1470-1477, 2020. https://doi.org/10.3201/eid2607.200282.

Sanders, J. M., Monogue, M. L., Jodlowski, T. Z., e Cutrell, J. B. Pharmacologic treatments for coronavirus disease 2019 (COVID-19): A review. JAMA, v. 323, n. 18, p. 1824-1836, 2020. https://doi.org/10.1001/jama.2020.6019. 
Siegfried, R. Modeling and Simulation of Complex Systems: A Framework for Efficient Agent-Based Modeling and Simulation. 1 ${ }^{\mathrm{a}}$ ed. Wiesbaden: Springer Vieweg, 2014.

Taylor, S., (ed.). Agent-based Modeling and Simulation. $1^{\mathrm{a}}$ ed. London: Palgrave Macmillan, 2014.

Valdez, L. D., Buono, C., Macri, P. A., e Braunstein, L. A. Social distancing strategies against disease spreading. Fractals, v. 21, n. 03n04, 1350019, 2013. https://doi.org/10.1142/S0218348X13500199.

Vynnycky, E. e White, R. G. An Introduction to Infectious Disease Modelling. $1^{\mathrm{a}}$ ed. EUA: Oxford University Press, 2010.

Wallinga, J. e Lipsitch, M. How generation intervals shape the relationship between growth rates and reproductive numbers. Proceedings of the Royal Society B: Biological Sciences, v. 274, n. 1609, p. 599-604, 2007. https://doi.org/10.1098/rspb.2006.3754.

Wolfram, C. An agent-based model of COVID-19. Complex Systems, v. 29, n. 1, p. 87-105, 2020. https://doi.org/10.25088/ComplexSystems.29.1.87.

Wu, J. T., Leung, K., Bushman, M., Kishore, N., Niehus, R., de Salazar, P. M., et al. Estimating clinical severity of COVID-19 from the transmission dynamics in Wuhan, China. Nature Medicine, v. 26, n. 4, p. 506-510, 2020. https://doi.org/10.1038/s41591-020-0822-7.

You, C., Deng, Y., Hu, W., Sun, J., Lin, Q., Zhou, F., et al. Estimation of the time-varying reproduction number of COVID-19 outbreak in China. International Journal of Hygiene and Environmental Health, v. 228, 113555, 2020. https://doi.org/10.1016/j.ijheh.2020.113555.

\section{Apêndices}

Os gráficos apresentados nesta seção correspondem ao comportamento, ao longo do tempo, dos cenários simulados, sendo, portanto, complementares aos dados apresentados nas Tabelas $3 \mathrm{e}$ 4. 
Figura 4: Curvas esperadas de difusão.

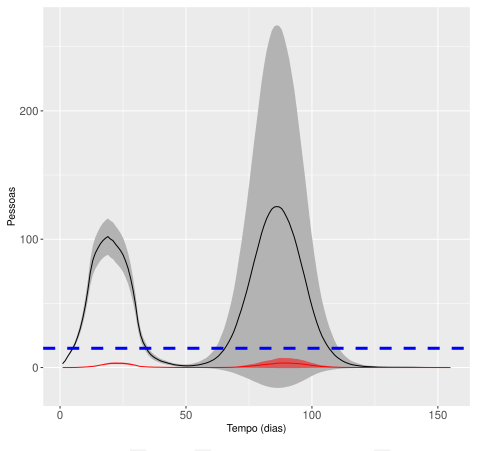

$A 30_{m} 10_{t} 30_{d}$
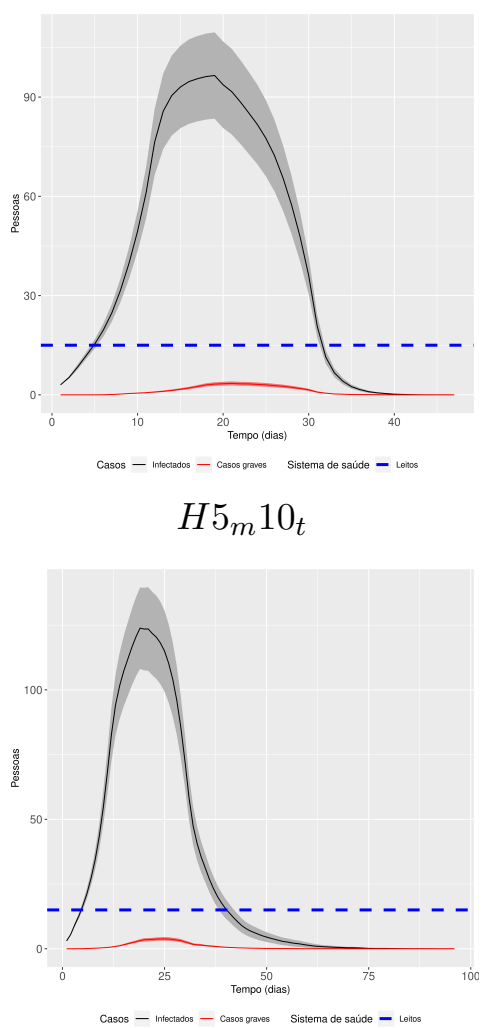

$H 30_{m} 10_{t}$

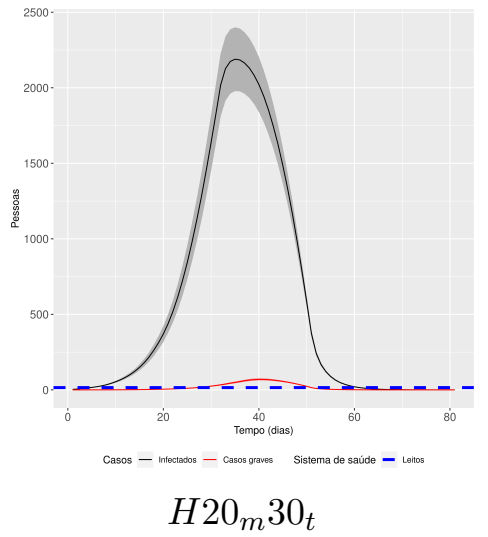

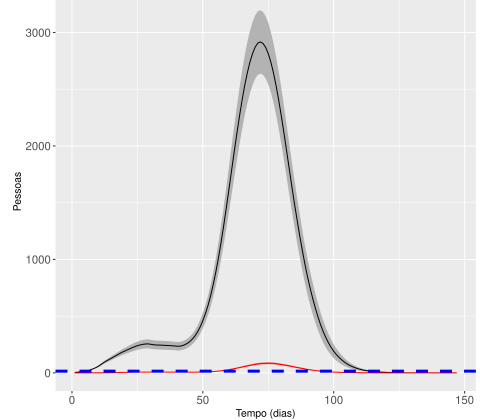
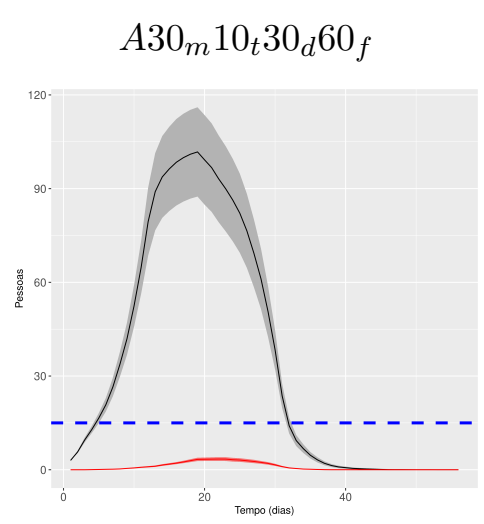

$H 10_{m} 10_{t}$
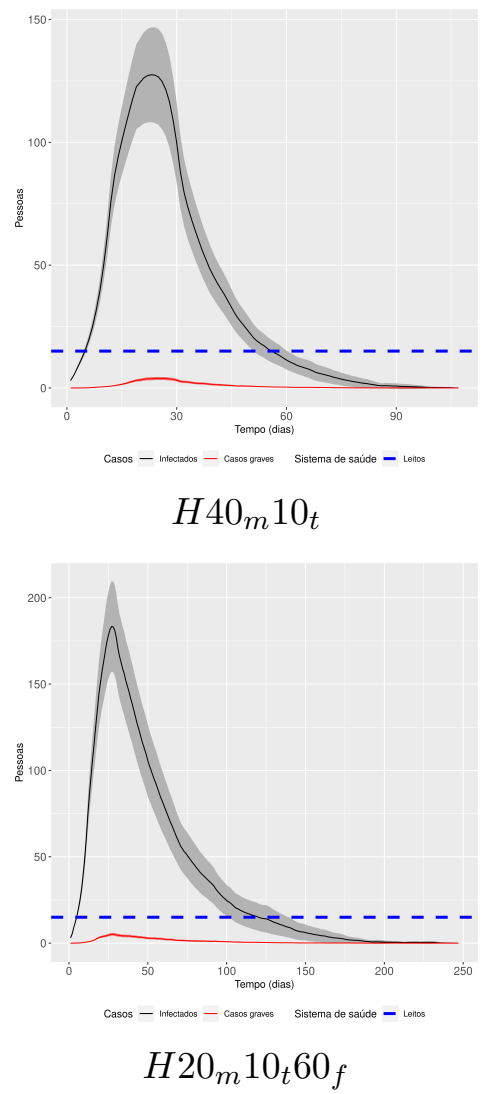

Fonte: Elaborado pelos autores.
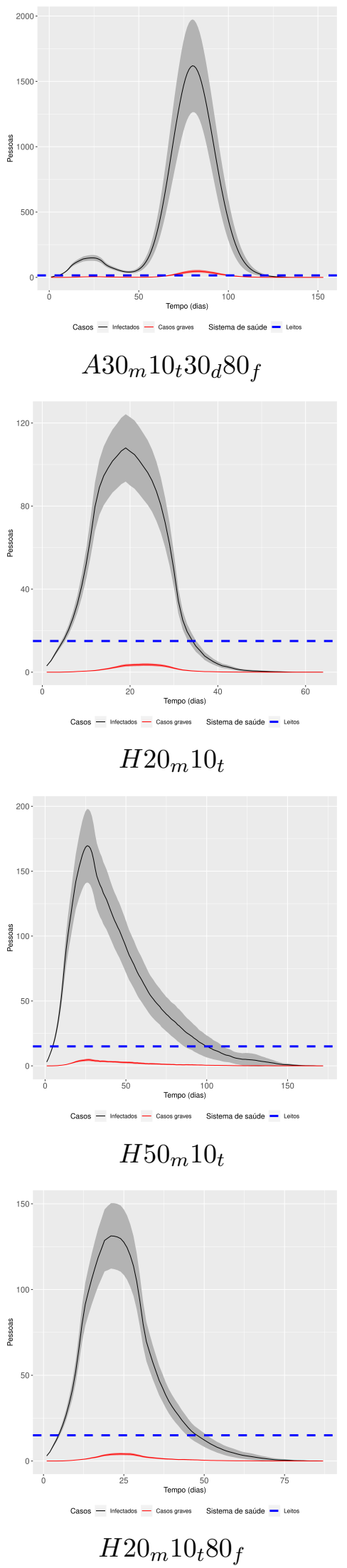
Figura 5: Curvas esperadas de difusão.
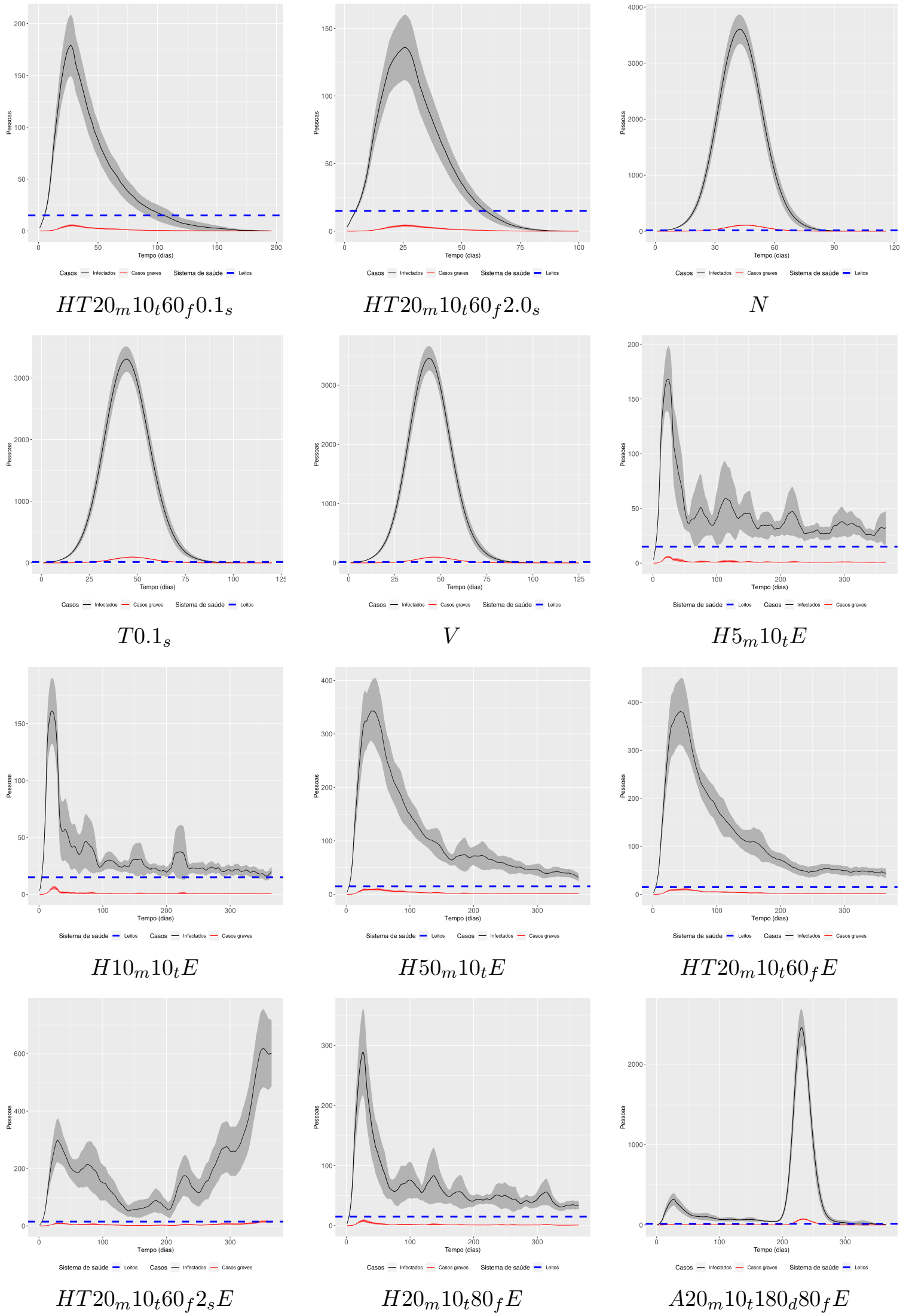

Fonte: Elaborado pelos autores. 
Figura 6: Curva esperada de difusão do cenário $N E$.

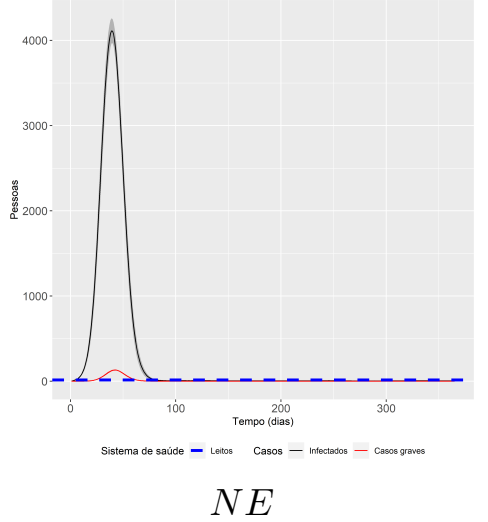

Fonte: Elaborado pelos autores. 